\title{
The application of FMEA to reduce defective rate from broken filament defects in the Direct Spin Drawing process
}

\author{
Parinee Kaewsom ${ }^{1}$, Napassavong Rojanarowan ${ }^{2}$ \\ ${ }^{I}$ (Department of Industrial Engineering, Faculty of Engineering, Chulalongkorn University, Bangkok, Thailand. \\ Parinee.k@student.chula.ac.th) \\ ${ }_{2}^{2}$ (Department of Industrial Engineering, Faculty of Engineering, Chulalongkorn University, Bangkok, Thailand. \\ Napassavong.o@chula.ac.th)
}

\begin{abstract}
This paper presents a methodology to reduce defective rate from broken filament defects in the Direct Spin Drawing process. The Failure Mode and Effects Analysis (FMEA)was used to analyze the causes of defect and prioritize those causes,leading to the pertinent improvement methods. The improvement method involved the development of better methods for detection of failures occurred in machine parts. After improvement, the defective rate from broken filament defects significantly was reduced from $3.35 \%$ to $1.76 \%$.
\end{abstract}

Keywords:-Broken filament, Defective Reduction, Direct Spin Draw process, Detection, FMEA

\section{INTRODUCTION}

Yarn is used in the manufacture of seat belt used in a vehicle. Yarn consists of many filaments. Broken filament was a major defect commonly found in producing the yarn. A sheet of yarn was considered as defective when there were two or more broken filaments per sheet. In the case study factory, the defective rate from broken filament defects was $3.35 \%$, which was $62.4 \%$ of the total defects. There were many possible factors, which can cause the broken filament defect. These factors were mainly machine-related. Thus, there was a need to identify the major root causes and solve them to obtain the lower defective rate.

\section{LITERATUREREVIEW}

The mechanical properties such as the stretch of the yarn, the tension of the yarn, and the friction between the yarn and the machine could cause the broken filament defect. The tension of the polyester yarn was related to the setting of machine factors such as the spinning temperature, the velocity at the spinneret outlet, the temperature of quench air, the velocity of quench air, and the spinning velocity [1]. The spin pack cleaning was important since a dirty spinneret hole will result in uneven polymeric liquid flow through the spinneret hole [2]. Furthermore, the parameters influencing the quality of the modern fiber draw process were the fiber radius,thedraw speed, and the draw tension [3]. Moreover, the parameters in the spinning and the drawing processes also affected the quality of the yarn. These parameters were the spinning speed, the spinning temperature, the speed of the Godet roller, the winder speed, and the steam pressure. The speed of the Godet roller directly affected the draw ratio, the tenacity, the elongation, and the shrinkage ratio [4]. There were many factors that could cause the broken filament defect. However, solving all the causes was not cost-effective. Thus, there is a need to prioritize the causes and improve on important factors. Thus, this research has the aim to investigate all possible factors in the Direct Spin Draw process and prioritize those causes to obtain important factors to be improved.

Failure Mode and Effect Analysis (FMEA) is a technique for evaluation of potential failure modes andtheir effects, the causes of the failure modes and its occurrence rates, and also the detection methods and their effectiveness. The consequences of FMEA development are to prevent or reduce the severity of failures, to detect the cause of failure by starting with highest-priority ones[5].A knitting company used FMEA as a tool to analyze the important causes and then to find solutions for further improvement[6].

\section{PRODUCTION PROCESS OF YARN}

The Direct Spin Drawing process (DSD) starts when the dry chip is melt into the polymeric liquid. Then, it is compressed through the spiral of the extruder. Next, the polymeric liquid flows through the heating box and the spin box, and then is compressed through the metering pump. After that the polymeric liquid is compressed through the spinneret holes to form the filaments. Next, the filaments are cooled by the quenching air and combined to form the yarn by a suction gun. Next, the yarn passes into the drawing zone, which helps stretch the yarn. In the drawing zone, the yarn passes through 5 Godet rollers. Each roller might have different temperature and speed. Between some of the rollers, there was an air guide to blow and form the yarn with 
pressure. After the yarn passes through all rollers, the yarn is rolled into a paper tube and is inspected before being sent to the customers.

\section{METHODOLOGY}

First the FMEA team was set up. Then, the team applied the concept of the criteria of FMEA to prioritize the causes to be improved. These criteria are the severity rating of the failure mode effects, the occurrence rating of failure causes, and the detection rating of the effectiveness of the detection methods [7].The FMEA criteria of AIAG [7] are commonly used in automotive and electronics industries. However, there is no FMEA criteria particularly developed for the textile industry. Thus, this research has developed the description of the FMEA score rating to be properly used in the case study company, which is in the textile industry. This research has developed the description of score rating for two criteria, which are the occurrence rating and the detection rating. The description of the severity rating was not developed since in this case, there is only one type of defect under consideration. The severity ratings of all failure modes are the same, which is 9 (filaments broke). The description of the developed score rating is shown in Table 1. Next, the team used the risk priority number (RPN), which is the multiplication of the severity rating, the occurrence rating, and the detection rating to prioritize the failure causes. The causes with higher RPNs were considered as the more important causes, which would be selected and solved further.

Table 1 The description of the developed occurrence rating and detecting rating

\begin{tabular}{|c|l|l|}
\hline Score & Occurrence Scale & \multicolumn{1}{c|}{ Detection Scale } \\
\hline 10 & Everyday & No inspection \\
\hline 9 & Every 3-6 days & Inspection when there is complaint \\
\hline 8 & Every 1-2 weeks & Inspection occasionally \\
\hline 7 & Every 3-4 weeks & Periodic visual inspection on some machines \\
\hline 6 & Every 1-2 months & Periodic visual inspection on all machines \\
\hline 5 & Every 3-6 month & Regular inspection with simple visual check \\
\hline 4 & Every 7-12 months & $\begin{array}{l}\text { Check with measuring instrument and monitor with control } \\
\text { chart. Then, take action when extreme point is found }\end{array}$ \\
\hline 3 & Every 1 - 2 years & $\begin{array}{l}\text { Check with measuring instrument and monitor with control } \\
\text { chart. Then, take action when sensitivity rules are violated }\end{array}$ \\
\hline 2 & Every 3 - 4 years & $100 \%$ inspection by automatic system with no alarm system \\
\hline 1 & More than 4 years & $100 \%$ inspection by automatic system with alarm system \\
\hline
\end{tabular}

\section{RESULTS}

After defining the criteria for FMEA analysis, then the team brainstormed to list out all potential failure modes, failure causes, and related detection methods according process functions. Then, the score ratings were given. Table 2 shows the score rating of 10 failure causes which have the highest RPNs. Out of these 10 failure causes, there are 8causes that were improved by changing the detection method or determining more appropriate detection frequency. The causes related to parameter setting were suggested to be studied further using the design of experiment technique to help find the optimal setting. 
The application of FMEA to reduce defective rate from broken filament defects in the Direct Spin

Table 2FMEAAnalysis and improvement solutions

\begin{tabular}{|c|c|c|c|c|c|c|c|c|c|c|c|c|c|c|c|c|}
\hline \multirow{2}{*}{$\begin{array}{l}\text { Process } \\
\text { Function/ } \\
\text { Requirem } \\
\text { ents }\end{array}$} & \multirow[b]{2}{*}{$\begin{array}{l}\text { Potential } \\
\text { Failure } \\
\text { Mode }\end{array}$} & \multirow{2}{*}{$\begin{array}{l}\text { Potential } \\
\text { Effect(s) of } \\
\text { Failure } \\
\text { Mode }\end{array}$} & \multirow[b]{2}{*}{$\mathrm{s}$} & \multirow{2}{*}{$\begin{array}{c}\text { Potential } \\
\text { Cause(s)/ } \\
\text { Mechanism(s) } \\
\text { of Failure }\end{array}$} & \multirow[b]{2}{*}{0} & \multicolumn{2}{|c|}{ Current Control } & \multirow[b]{2}{*}{$\mathrm{D}$} & \multirow[b]{2}{*}{$\begin{array}{l}\mathrm{R} \\
\mathrm{P} \\
\mathrm{N}\end{array}$} & \multirow[b]{2}{*}{$\begin{array}{l}\text { Recommended } \\
\text { Action(s) }\end{array}$} & \multirow{2}{*}{$\begin{array}{c}\text { Responsibility } \\
\text { \&Target } \\
\text { Completion } \\
\text { Date }\end{array}$} & \multicolumn{5}{|c|}{ Actions Result } \\
\hline & & & & & & Prevention & Detection & & & & & $\begin{array}{l}\text { Actions } \\
\text { Taken }\end{array}$ & $\mathrm{s}$ & 0 & $\mathrm{D}$ & \\
\hline \multirow[t]{3}{*}{$\begin{array}{l}\text { Air Guide } \\
\text { shakes off } \\
\text { oil from } \\
\text { yam and } \\
\text { form yam } \\
\text { together }\end{array}$} & $\begin{array}{c}\text { Yam } \\
\text { position } \\
\text { in Air } \\
\text { Guide } \\
\text { holes is } \\
\text { not } \\
\text { centered. }\end{array}$ & $\begin{array}{l}\text { Yam swings } \\
\text { and scratches } \\
\text { with Air } \\
\text { Guide, } \\
\text { leading to } \\
\text { broken yam. }\end{array}$ & 9 & $\begin{array}{l}\text { Nuts of Air } \\
\text { guide are } \\
\text { loosening. }\end{array}$ & 9 & - & $\begin{array}{c}\text { Inspect } \\
\text { position of } \\
\text { yam in Air } \\
\text { Guide holes } \\
\text { when there is } \\
\text { complaint }\end{array}$ & 9 & $\begin{array}{l}7 \\
2 \\
9\end{array}$ & $\begin{array}{l}\text { Check nuts every } \\
2 \text { days and check } \\
\text { the center of yam } \\
\text { every shift. }\end{array}$ & $\begin{array}{c}\text { Process } \\
\text { Engineer } \\
(20 / 7 / 2013)\end{array}$ & $\begin{array}{c}\text { Check nuts } \\
\text { every } 2 \text { days } \\
\text { and check } \\
\text { the center of } \\
\text { yam every } \\
\text { shift. }\end{array}$ & 9 & 6 & 5 & 2 \\
\hline & \multirow[t]{2}{*}{$\begin{array}{c}\text { The } \\
\text { balloon } \\
\text { of yam is } \\
\text { bad. }\end{array}$} & \multirow[t]{2}{*}{$\begin{array}{l}\text { Yam has bad } \\
\text { balloon. } \\
\text { Then, it } \\
\text { swings and } \\
\text { scratches } \\
\text { with Air } \\
\text { Guide, } \\
\text { leading to } \\
\text { broken yam. }\end{array}$} & 9 & $\begin{array}{l}\text { Holes of Air } \\
\text { Guide are dirty. }\end{array}$ & 8 & $\begin{array}{l}\text { Holes of Air } \\
\text { guide are } \\
\text { inspected } \\
\text { longer than } \\
\text { once a week } \\
\text { by visual } \\
\text { check. }\end{array}$ & $\begin{array}{c}\text { Inspect } \\
\text { balloon of } \\
\text { yam when } \\
\text { there is } \\
\text { complaint }\end{array}$ & 9 & $\begin{array}{l}6 \\
4 \\
8\end{array}$ & $\begin{array}{l}\text { Clean Air Guide } \\
\text { every } 3 \text { months. }\end{array}$ & $\begin{array}{l}\text { Process } \\
\text { Engineer } \\
(20 / 7 / 2013)\end{array}$ & $\begin{array}{l}\text { Clean Air } \\
\text { guide every } \\
3 \text { months } \\
\text { and check } \\
\text { the } \\
\text { cleanliness } \\
\text { of Air Guide } \\
\text { hole before } \\
\text { using }\end{array}$ & 9 & 5 & 5 & 2 \\
\hline & & & 9 & $\begin{array}{l}\text { Regulator is } \\
\text { ruined. }\end{array}$ & 8 & - & $\begin{array}{c}\text { Inspect } \\
\text { balloon of } \\
\text { yam when } \\
\text { there is } \\
\text { complaint }\end{array}$ & 9 & $\begin{array}{l}6 \\
4 \\
8\end{array}$ & $\begin{array}{l}\text { Check Air Guide } \\
\text { pressure every } \\
\text { shift and check } \\
\text { regulator gauge } \\
\text { when coating. }\end{array}$ & $\begin{array}{l}\text { Supervisor } \\
(20 / 7 / 2013)\end{array}$ & $\begin{array}{l}\text { Check Air } \\
\text { Guide } \\
\text { pressure } \\
\text { every shift } \\
\text { and check } \\
\text { regulator } \\
\text { gauge when } \\
\text { coating. }\end{array}$ & 9 & 7 & 5 & $\begin{array}{l}3 \\
1 \\
5\end{array}$ \\
\hline
\end{tabular}

Table 2FMEA Analysis and improvement solutions (Cont.)

\begin{tabular}{|c|c|c|c|c|c|c|c|c|c|c|c|c|c|c|c|c|}
\hline \multirow{2}{*}{$\begin{array}{l}\text { Process } \\
\text { Function/ } \\
\text { Requirem } \\
\text { ents }\end{array}$} & \multirow{2}{*}{$\begin{array}{c}\text { Potential } \\
\text { Failure } \\
\text { Mode }\end{array}$} & \multirow{2}{*}{$\begin{array}{l}\text { Potential } \\
\text { Effect(s) of } \\
\text { Failure }\end{array}$} & \multirow[b]{2}{*}{$\mathrm{s}$} & \multirow{2}{*}{$\begin{array}{c}\text { Potential } \\
\text { Cause(s)/ } \\
\text { Mechanism(s) } \\
\text { of Failure }\end{array}$} & \multirow[b]{2}{*}{0} & \multicolumn{2}{|c|}{ Current Control } & \multirow[b]{2}{*}{$\mathrm{D}$} & \multirow[b]{2}{*}{$\begin{array}{l}\mathrm{R} \\
\mathrm{P} \\
\mathrm{N}\end{array}$} & \multirow[b]{2}{*}{$\begin{array}{l}\text { Recommended } \\
\text { Action(s) }\end{array}$} & \multirow{2}{*}{$\begin{array}{c}\text { Responsibility } \\
\text { \&Target } \\
\text { Completion } \\
\text { Date }\end{array}$} & \multicolumn{5}{|c|}{ Actions Result } \\
\hline & & & & & & Prevention & Detection & & & & & $\begin{array}{l}\text { Actions } \\
\text { Taken }\end{array}$ & $\mathrm{s}$ & 0 & $\mathrm{D}$ & $\begin{array}{l}\mathrm{R} \\
\mathrm{P}\end{array}$ \\
\hline \multirow[t]{4}{*}{$\begin{array}{c}\text { Godet } \\
\text { Roller } \\
\text { (GR) } \\
\text { stretches } \\
\text { yam and } \\
\text { guide yam } \\
\text { through } \\
\text { yam path. }\end{array}$} & $\begin{array}{l}\text { Yam is } \\
\text { too tight } \\
\text { or too } \\
\text { slack. }\end{array}$ & $\begin{array}{l}\text { Too tight yam } \\
\text { is easily } \\
\text { broken. Too } \\
\text { slack yam can } \\
\text { scratch with } \\
\text { machine parts, } \\
\text { leading to } \\
\text { broken yam. }\end{array}$ & 9 & $\begin{array}{l}\text { Inappropriate } \\
\text { speed setting. }\end{array}$ & 8 & $\begin{array}{c}\text { Recheck } \\
\text { speed setting } \\
\text { every } 8 \text { hours. }\end{array}$ & $\begin{array}{l}\text { Inspect yam } \\
\text { occasionally }\end{array}$ & 8 & $\begin{array}{l}5 \\
7 \\
6\end{array}$ & $\begin{array}{l}\text { Perform design of } \\
\text { experiment to test } \\
\text { and find } \\
\text { appropriate speed } \\
\text { setting. }\end{array}$ & $\begin{array}{l}\text { Production } \\
\text { Engineer } \\
(28 / 7 / 2013)\end{array}$ & $\begin{array}{l}\text { Perform } \\
\text { design of } \\
\text { experiment } \\
\text { to test and } \\
\text { find } \\
\text { appropriate } \\
\text { speed setting } \\
\text { for each } \\
\text { Godet roller. }\end{array}$ & 9 & 7 & 3 & $\begin{array}{l}1 \\
8 \\
9\end{array}$ \\
\hline & $\begin{array}{l}\text { Yam is } \\
\text { too soft. }\end{array}$ & $\begin{array}{l}\text { Too soft yam } \\
\text { stretches less } \\
\text { and difficult } \\
\text { to guide, } \\
\text { leading to } \\
\text { broken yam. }\end{array}$ & 9 & $\begin{array}{l}\text { Inappropriate } \\
\text { temperature } \\
\text { setting. }\end{array}$ & 8 & $\begin{array}{c}\text { Recheck } \\
\text { temperature } \\
\text { setting every } \\
8 \text { hours. }\end{array}$ & $\begin{array}{l}\text { Inspect yam } \\
\text { quality } \\
\text { occasionally }\end{array}$ & 8 & $\begin{array}{l}5 \\
7 \\
6\end{array}$ & $\begin{array}{l}\text { Perform design of } \\
\text { experiment to test } \\
\text { and find } \\
\text { appropriate } \\
\text { temperature } \\
\text { setting. }\end{array}$ & $\begin{array}{l}\text { Production } \\
\text { Engineer } \\
(28 / 7 / 2013)\end{array}$ & $\begin{array}{l}\text { Perform } \\
\text { design of } \\
\text { experiment } \\
\text { to test and } \\
\text { find } \\
\text { appropriate } \\
\text { temperature } \\
\text { setting for } \\
\text { GR1, GR2. }\end{array}$ & 9 & 7 & 3 & $\begin{array}{l}1 \\
8 \\
9\end{array}$ \\
\hline & $\begin{array}{l}\text { Godet } \\
\text { Roller is } \\
\text { cracked } \\
\text { or } \\
\text { scratched } \\
\text {. }\end{array}$ & $\begin{array}{l}\text { Yam passes } \\
\text { through } \\
\text { cracked or } \\
\text { scratched } G R \\
\text { surface, } \\
\text { leading to } \\
\text { broken yam. }\end{array}$ & 9 & $\begin{array}{c}\text { Yam always } \\
\text { passes through } \\
\text { the same } \\
\text { position on GR. }\end{array}$ & 6 & $\begin{array}{c}\text { Check the GR } \\
\text { surface longer } \\
\text { than once a } \\
\text { month }\end{array}$ & $\begin{array}{c}\text { Inspect GR } \\
\text { surface } \\
\text { occasionally }\end{array}$ & 8 & $\begin{array}{l}4 \\
3 \\
2\end{array}$ & $\begin{array}{c}\text { Create standard } \\
\text { for checking GR } \\
\text { surface every } 2 \\
\text { weeks and } \\
\text { changing the GR. }\end{array}$ & $\begin{array}{l}\text { Production } \\
\text { Engineer } \\
(28 / 7 / 2013)\end{array}$ & $\begin{array}{c}\text { Create } \\
\text { standard for } \\
\text { checking GR } \\
\text { surface } \\
\text { every } 2 \\
\text { weeks and } \\
\text { changing the } \\
\text { GR. }\end{array}$ & 9 & 6 & 7 & $\begin{array}{l}3 \\
7 \\
8\end{array}$ \\
\hline & $\begin{array}{l}\text { Godet } \\
\text { roller is } \\
\text { dirty. }\end{array}$ & $\begin{array}{l}\text { Yarn which } \\
\text { passes } \\
\text { through dirt } \\
\text { gets stuck and } \\
\text { scratched, } \\
\text { leading to } \\
\text { broken yam. }\end{array}$ & 9 & $\begin{array}{c}\text { Cleaning } \\
\text { method is } \\
\text { imeffective and } \\
\text { cleaning } \\
\text { frequency is not } \\
\text { appropriate. }\end{array}$ & 9 & $\begin{array}{c}\text { Check the } \\
\text { cleanliness of } \\
\text { GR every day } \\
\text { but no criteria } \\
\text { for deciding } \\
\text { whether the } \\
\text { roller is clean } \\
\text { or not. }\end{array}$ & $\begin{array}{l}\text { Inspect the } \\
\text { cleanliness of } \\
\text { GR when } \\
\text { there is } \\
\text { complaint }\end{array}$ & 9 & $\begin{array}{l}7 \\
2 \\
9\end{array}$ & $\begin{array}{l}\text { Create photos of } \\
\text { GR surface before } \\
\text { - after cleaning } \\
\text { and use it as a } \\
\text { standard for } \\
\text { inspection. }\end{array}$ & $\begin{array}{l}\text { Production } \\
\text { Engineer } \\
(28 / 7 / 2013)\end{array}$ & $\begin{array}{c}\text { Create } \\
\text { photos of } \\
\text { GR surface } \\
\text { before - } \\
\text { after } \\
\text { cleaning and } \\
\text { use it as a } \\
\text { standard for } \\
\text { inspection. }\end{array}$ & 9 & 8 & 5 & $\begin{array}{l}3 \\
6 \\
0\end{array}$ \\
\hline
\end{tabular}


The application of FMEA to reduce defective rate from broken filament defects in the Direct Spin

Table 2FMEA Analysis and improvement solutions (Cont.)

\begin{tabular}{|c|c|c|c|c|c|c|c|c|c|c|c|c|c|c|c|c|}
\hline \multirow{2}{*}{$\begin{array}{c}\text { Process } \\
\text { Function/ } \\
\text { Requirem } \\
\text { ents }\end{array}$} & \multirow{2}{*}{$\begin{array}{l}\text { Potential } \\
\text { Failure } \\
\text { Mode }\end{array}$} & \multirow{2}{*}{$\begin{array}{l}\text { Potential } \\
\text { Effect(s) of } \\
\text { Failure }\end{array}$} & \multirow[b]{2}{*}{$\mathrm{s}$} & \multirow{2}{*}{\begin{tabular}{|c|} 
Potential \\
Cause(s)/ \\
Mechanism(s) \\
of Failure
\end{tabular}} & \multirow[b]{2}{*}{0} & \multicolumn{2}{|c|}{ Current Control } & \multirow[b]{2}{*}{$\mathrm{D}$} & \multirow{2}{*}{$\begin{array}{l}\mathrm{R} \\
\mathrm{P} \\
\mathrm{N}\end{array}$} & \multirow[b]{2}{*}{$\begin{array}{l}\text { Recommended } \\
\text { Action(s) }\end{array}$} & \multirow{2}{*}{$\begin{array}{c}\text { Responsibility } \\
\text { \&Target } \\
\text { Completion } \\
\text { Date }\end{array}$} & \multicolumn{5}{|c|}{ Actions Result } \\
\hline & & & & & & Prevention & Detection & & & & & $\begin{array}{l}\text { Actions } \\
\text { Taken }\end{array}$ & $\mathrm{S}$ & 0 & $\mathrm{D}$ & $\begin{array}{l}R \\
P\end{array}$ \\
\hline \multirow[t]{3}{*}{$\begin{array}{l}\text { Winder } \\
\text { spins yam } \\
\text { into tube. }\end{array}$} & $\begin{array}{l}\text { Hanetora } \\
\text { and } \\
\text { Wave } \\
\text { guide are } \\
\text { in wrong } \\
\text { position. }\end{array}$ & $\begin{array}{l}\text { Yam scratches } \\
\text { with Hanetora } \\
\text { and Wave } \\
\text { guide, leading } \\
\text { to broken } \\
\text { yam. }\end{array}$ & 9 & $\begin{array}{c}\text { Broken } \\
\text { filament on } \\
\text { yam made } \\
\text { Hanetora and } \\
\text { Wave guide } \\
\text { move. }\end{array}$ & 7 & - & $\begin{array}{c}\text { Check the } \\
\text { position of } \\
\text { Hanetora and } \\
\text { Wave guide } \\
\text { when there is } \\
\text { complaint }\end{array}$ & 9 & \begin{tabular}{l|}
5 \\
6 \\
7
\end{tabular} & $\begin{array}{c}\text { Check the } \\
\text { position of } \\
\text { Hanetora and } \\
\text { Wave guide every } \\
2 \text { weeks }\end{array}$ & $\begin{array}{l}\text { Maintenance } \\
(10 / 7 / 2013)\end{array}$ & $\begin{array}{l}\text { Check the } \\
\text { position of } \\
\text { Hanetora and } \\
\text { Wave guide } \\
\text { every } 2 \\
\text { weeks. }\end{array}$ & 9 & 7 & 6 & 3 \\
\hline & $\begin{array}{l}\text { Roller } \\
\text { Bail is } \\
\text { scratched }\end{array}$ & $\begin{array}{l}\text { Yam rubs } \\
\text { with scratched } \\
\text { Roller Bail, } \\
\text { leading to } \\
\text { broken yam. }\end{array}$ & 9 & $\begin{array}{l}\text { Cutter which } \\
\text { is used to cut } \\
\text { broken } \\
\text { filament } \\
\text { scratches on } \\
\text { the Roller } \\
\text { Bail. }\end{array}$ & 7 & - & $\begin{array}{c}\text { Periodically } \\
\text { visual inspect } \\
\text { Roller Bail } \\
\text { surface of all } \\
\text { machines } \\
\text { every } 2-7 \\
\text { days. }\end{array}$ & 6 & \begin{tabular}{l|}
3 \\
7 \\
8
\end{tabular} & $\begin{array}{l}\text { - Use heater cutter } \\
\text { instead. } \\
\text { - Make standard } \\
\text { for checking } \\
\text { scratch and check } \\
\text { every } 2 \text { weeks to } \\
\text { help decide on } \\
\text { Roller Bail } \\
\text { changing time. }\end{array}$ & $\begin{array}{c}\text { Maintenance } \\
(10 / 7 / 2013)\end{array}$ & $\begin{array}{l}\text { - Use heater } \\
\text { cutter instead. } \\
\text { - Make } \\
\text { standard for } \\
\text { checking } \\
\text { scratch and } \\
\text { check every } 2 \\
\text { weeks. }\end{array}$ & 9 & 8 & 5 & 3 \\
\hline & $\begin{array}{l}\text { Hanetora } \\
\text { and } \\
\text { Wave } \\
\text { guide } \\
\text { surface } \\
\text { are } \\
\text { scratched }\end{array}$ & $\begin{array}{l}\text { Yam rubs } \\
\text { with scratched } \\
\text { Roller Bail, } \\
\text { leading to } \\
\text { broken yam. }\end{array}$ & 9 & $\begin{array}{l}\text { Hanetora and } \\
\text { Wave guide } \\
\text { are used for a } \\
\text { long time. }\end{array}$ & 6 & - & $\begin{array}{c}\text { Periodically } \\
\text { visual inspect } \\
\text { Roller Bail } \\
\text { surface of all } \\
\text { machines } \\
\text { every } 10-14 \\
\text { days. }\end{array}$ & 6 & $\begin{array}{l}3 \\
2 \\
4\end{array}$ & $\begin{array}{l}\text { Make standard for } \\
\text { checking scratch } \\
\text { and check every } 2 \\
\text { weeks to help } \\
\text { decide on } \\
\text { Hanetora and } \\
\text { Wave guide } \\
\text { changing time. }\end{array}$ & $\begin{array}{l}\text { Maintenance } \\
(10 / 7 / 2013)\end{array}$ & $\begin{array}{c}\text { Make } \\
\text { standard for } \\
\text { checking } \\
\text { scratch and } \\
\text { check every } 2 \\
\text { weeks. }\end{array}$ & 9 & 6 & \begin{tabular}{|l|}
5 \\
\end{tabular} & \\
\hline
\end{tabular}

\section{SUMMARY}

This paper presents a methodology to reduce the defective rate from broken filament defects in the Direct Spin Drawing process by using the Failure Mode and Effects Analysis (FMEA) technique. FMEA helps analyze and prioritize the important failure causes to be improved further. After the FMEA analysis, the selected causes were grouped into two groups.Group 1 involves the causes, which were improved by the changing the detection methods or determining more appropriate detection frequency. Group 2involves the causes related to the machine parameter setting, which suggested to be further improved by the use of the design of experiment technique.After improving the detection method, the defective rate from broken filament defects was reduced from $3.35 \%$ to $1.76 \%$.

\section{REFERENCES}

[1] H. Liqian, W. Xueli, Y. Jianyong, and W. Baojian, "Numerical Simulation on Melt Spinning of One-step Process POY/FDY Polyester Combined Yarn," The 7th International Conference on Computer Science \& Education, pp. 412-414, July 14-17 2012.

[2] J. Hui, Z. Yue-ling, and W. Da-zhi, "Optimal Scheduling for the Cleaning Spin Pack in Polyester Production," Ninth International Conference on Hybrid Intelligent Systems, pp. 103-107, 2009.

[3] K.-M. Lee, Z. Wei, Z. Zhou, S.-P. Hong, Fellow, and IEEE, "Computational Thermal Fluid Models for Design of a Modern Fiber Draw Process," IEEE Transaction on automation Science and Engineering, vol. 3, pp. 108-118, 1 January 2006.

[4] Y.-S. Ho, H.-Y. Kim, F.-L. Jin, and S.-J. Park, "Effects of Spinning Conditions on Properties of Polyester Yarn Prepared using an Ultra-high-speed Melt Spinning Technique Equipped with a Steam Chamber," Bull. Korean Chem. Soc., vol. 31, pp. 3252-3258, 31 November 2010.

[5] S.N.Gaikwad and M.M.Mulkutkar, "Reliability based design with FMEA AND FTA," IOSR Journal of Mechanical and Civil Engineering (IOSR-JMCE), pp. 21-25.

[6] V. Ozyazgan and F. Z. Engin, "FMEA analysis and applications in knitting industry," TekstilveKonfeksiyon, vol. 23, pp. 228-232, 2013.

[7] A. I. A. Group, Potential Failure Mode and Effects Analysis, 3 ed. Michigan, USA, 2001. 